\title{
RELATIVE SUPERIOR MANDELBROT SETS AND RELATIVE SUPERIOR JULIA SETS FOR INTEGER AND NON-INTEGER VALUES
}

\author{
Rajeshri Rana ${ }^{1}$, Yashwant Singh Chauhan ${ }^{2}$ \\ ${ }^{1}$ Assistant Professor, Mathematics, ${ }^{2}$ Assistant Professor, CSED/MCA, G. B. Pant Engineering College, Pauri-Garhwal, \\ Uttarakhand, India,ranarajeshri@rediffmail,yashwant.s.chauhan@gmail.com \\ Abstract
}

The fractals generated from the self-squared function, $z \rightarrow z^{2}+c$ where $z$ and care complex quantities have been studied extensively in the literature. This paper studies the transformation of the function $z \rightarrow z^{n}+c, n \geq 2$ and analyzed the $z$ plane and $c$ plane fractal images generated from the iteration of these functions using Ishikawa iteration for integer and non-integer values. Also, we explored the drastic changes that occurred in the visual characteristics of the images from $n=$ integer value to $n=$ non integer value.

Keywords: Complex dynamics, Relative Superior Julia set, Relative Superior Mandelbrot set.

\section{INTRODUCTION}

"Fractal" is a word invented by Mandelbrot to bring together under the one heading, a large class of objects that have played an historical role in the development of pure mathematics. A great revolution of ideas separates the classical mathematics of the 19th century from the modern mathematics of the 20th. Classical mathematics had its roots in the regular geometric structures of Euclid and the continuously evolving dynamics of Newton. Modern mathematics began with Cantor's set theory and Piano's space-filling curve. Historically, the revolution was forced by the discovery of mathematical structures that did not fit the patterns of Euclid and Newton. These new structures were regarded as 'pathological,'... as a 'gallery of monsters,' kin to the cubist painting and atonal music that were upsetting established standards of taste in the arts at about the same time. The mathematicians who created the monsters regarded them as important in showing that the world of pure mathematics contains a richness of possibilities going far beyond the simple structures that they saw in Nature. Twentieth-century mathematics flowered in the belief that it had transcended completely the limitations imposed by its natural origins.

Perhaps the Mandelbrot set [12] is the most popular object in fractal theory. It is believed that it is not only the most beautiful object, which has been made visible but the most complex also. This object was given by Benoit B. Mandelbrot in 1979 and has been the subject of intense research right from its advent. It is known to us that all the complex quadratic functions are topologically conjugate to the complex quadratic function $\mathrm{Q}(\mathrm{z})=\mathrm{z} 2+\mathrm{c}$. Recall that every Julia set for $\mathrm{Q}(\mathrm{z})=\mathrm{z} 2$ $+\mathrm{c}$ is either connected or totally disconnected. The Mandelbrot set works as a locator for the two types of Julia sets. Each point in the Mandelbrot set represents a c-value for which the Julia set is connected and each point in its complement represents a c-value for which the Julia set is totally disconnected [12].

The fractals generated from the self-squared function, $z \rightarrow z^{2}+c$ where $z$ and $c$ are complex quantities, have been studied extensively in the literature[5, 6, 7,8 \& 11]. Recently, the generalized transformation function $z \rightarrow z^{-n}+c$ for positive integer values of $n$ has been considered by K. W. Shirriff [11]. The z plane fractal images for the function $z_{n+1} \leftarrow z_{n}^{\alpha}+c$ for positive and negative, both integer and non-integer values of $n$ have been presented by Gujar et al. along with some conjectures about their visual characteristics[6, 7]. In this paper, we have considered the transformation of the function $z \rightarrow z^{n}+c, n \geq 2$ and analyzed the $z$ plane and $c$ plane fractal images generated from the iteration of these functions using Ishikawa iteration for integer and non-integer values. Also, we explored the drastic changes that occurred in the visual characteristics of the images from $n=$ integer value to $n=$ non integer value[2, $3 \& 15]$.

\section{PRELIMINARIES}

Let $\left\{z_{n}: n=1,2,3,4 \ldots \ldots \ldots\right\}$, denoted by $\left\{z_{n}\right\}$ be a
sequence of complex numbers. Then, we say $\operatorname{Lim}_{n \rightarrow \infty} z_{n}=\infty$ if, for given $M>0$, there exists $N>0$, such that for all $n>N$, we 
must have $\left|z_{n}\right|>M$. Thus all the values of ${ }^{z_{n}}$, lies outside a circle of radius $M$, for sufficiently large values of $n$.

Let $Q(z)=a_{0} z^{n}+a_{1} z^{n-1}+a_{2} z^{n-2}+\ldots \ldots \ldots \ldots . .+a_{n-1} z^{1}+a_{n} z^{0} ; a_{0} \neq 0$ be a polynomial of degree $\mathrm{n}$, where $n \geq 2$. The coefficients are allowed to be complex numbers. In other words, it follows that $Q_{c}(z)=z^{2}+c$

Definition 2.1[4]:Let $X$ be a nonempty set and $f: X \rightarrow X$. For any point $x_{0} \in X$, the Picard's orbit is defined as the set of iterates of a point ${ }^{x_{0}}$, that is; $O\left(f, x_{0}\right)=\left\{x_{n} ; x_{n}=f\left(x_{n-1}\right), n=1,2,3 \ldots ..\right\}$.

In functional dynamics, we have existence of two different types of points. Points that leave the interval after a finite number are in stable set of infinity. Points that never leave the interval after any number of iterations have bounded orbits. So, an orbit is bounded if there exists a positive real number, such that the modulus of every point in the orbit is less than this number.

The collection of points that are bounded, i.e. there exists $\mathrm{M}$, such that $\left|Q^{n}(z)\right| \leq M$, for all $\mathrm{n}$, is called as a prisoner set while the collection of points that are in the stable set of infinity is called the escape set. Hence, the boundary of the prisoner set is simultaneously the boundary of escape set and that is Mandelbrot set for $\mathrm{Q}$.

Definition 2.2[4]:The Mandelbrot set $M$ for the quadratic $Q_{c}(z)=z^{2}+c$ is defined as the collection of all $c \in C_{\text {for }}$ which the orbit of the point 0 is bounded, that is $M=\left\{c \in C:\left\{Q_{c}^{n}(0)\right\} ; n=0,1,2, \ldots . . . i s\right.$ bounded $\}$. An equivalent formulation is $M=\left\{c \in C:\left\{Q_{c}^{n}(0)\right.\right.$ does not tend to $\infty$ as $\left.n \rightarrow \infty\right\}$. We choose the initial point 0 , as 0 is the only critical point of $Q_{c}$.

\section{ISHIKAWA ITERATION FOR RELATIVE SUPERIOR MANDELBROT SETS AND RELATIVE SUPERIOR JULIA SETS}

Let $\mathrm{X}$ be a subset of real or complex numbers and $f: X \rightarrow X$. For $x_{0} \in X$, we construct the sequences $\left\{x_{n}\right\}$ and $\left\{y_{n}\right\}$ in $X$ in the following manner:

$$
\begin{aligned}
& y_{0}=s_{0}^{\prime} f\left(x_{0}\right)+\left(1-s_{0}^{\prime}\right) x_{0} \\
& y_{1}=s_{1}^{\prime} f\left(x_{1}\right)+\left(1-s_{1}^{\prime}\right) x_{1} \ldots \\
& y_{n}=s_{n}^{\prime} f\left(x_{n}\right)+\left(1-s_{n}^{\prime}\right) x_{n}
\end{aligned}
$$

where $0 \leq s_{n}^{\prime} \leq 1$ and $\left\{s_{n}^{\prime}\right\}$ is convergent to non zero number and

$$
\begin{aligned}
& x_{1}=s_{0} f\left(y_{0}\right)+\left(1-s_{0}\right) x_{0} \\
& x_{2}=s_{1} f\left(y_{1}\right)+\left(1-s_{1}\right) x_{1} \ldots \\
& x_{n}=s_{n-1} f\left(y_{n-1}\right)+\left(1-s_{n-1}\right) x_{n-1}
\end{aligned}
$$

where $0 \leq s_{n} \leq 1$ and $\left\{s_{n}\right\}_{\text {is convergent to non zero }}$ number[9].

Definition 3.1[2,15]: The sequences $\left\{x_{n}\right\}$ and $\left\{y_{n}\right\}$ constructed above is called Ishikawa sequences of iterations or relative superior sequences of iterates. We denote it by $R S O\left(x_{0}, s_{n}, s_{n}^{\prime}, t\right)$.

Notice that $\operatorname{RSO}\left(x_{0}, s_{n}, s_{n}^{\prime}, t\right) \quad$ with $\quad s_{n}^{\prime}=1 \quad$ is $R S O\left(x_{0}, s_{n}, t\right)$ i.e. Mann's orbit and if we place $s_{n}=s_{n}^{\prime}=1$ then $R S O\left(x_{0}, s_{n}, s_{n}^{\prime}, t\right)$ reduces to $O\left(x_{0}, t\right)$.

$$
\text { We remark that Ishikawa orbit }
$$
$R S O\left(x_{0}, s_{n}, s_{n}^{\prime}, t\right)$ with $s_{n}^{\prime}=1 / 2$ is Relative Superior orbit. Now we define Mandelbrot sets for function with respect to Ishikawa iterates. We call them as Relative Superior Mandelbrot sets.

Definition 3.2[15]: Relative Superior Mandelbrot set SM for the function of the form $Q_{c}(z)=z^{n}+c$, where $\mathrm{n}=1,2,3$, $4 \ldots$ is defined as the collection of $c \in C$ for which the orbit of 0 is bounded i.e.

$R S M=\left\{c \in C: Q_{c}^{k}(0): k=0,1,2 \ldots\right\}$ is bounded.

Definition 3.3[2]: The set of points SK whose orbits are bounded under Relative superior iteration of function $\mathrm{Q}(\mathrm{z})$ is called Relative Superior Julia sets. Relative Superior Julia set of Q is boundary of Julia set RSK.

3.4 Escape Criterion[2,15]: Fractals have been generated from $z \rightarrow z^{n}+c, n \geq 2$ and $z \rightarrow\left(z^{n}+c\right)^{-1}, \quad n \geq 2$ using escape-time techniques, for example by Gujar etal.[6, 7] 
and Glynn [8]. We have used in this paper escape time criteria of Relative Superior Ishikawa iterates for both of these functions.

Escape Criterion for Quadratics: Suppose that $|z|>\max \left\{|c|, 2 / s, 2 / s^{\prime}\right\}$, then $\left|z_{n}\right|>(1+\lambda)^{n}|z|$ and $\left|z_{n}\right| \rightarrow \infty$ as $n \rightarrow \infty$.So, $\quad|z| \geq|c|_{\text {and }} \quad|z|>2 / s_{\text {as }} \quad$ well as $|z|>2 / s^{\prime}$ shows the escape criteria for quadratics.

$\begin{array}{llr}\text { Escape Criterion for Cubics: } & \text { Suppose } \\ |z|>\max \left\{|b|,(|a|+2 / s)^{1 / 2},\left(|a|+2 / s^{\prime}\right)^{1 / 2}\right\} & \text { then }\left|z_{n}\right| \rightarrow \infty\end{array}$ as $n \rightarrow \infty$. This gives an escape criterion for cubic polynomials.

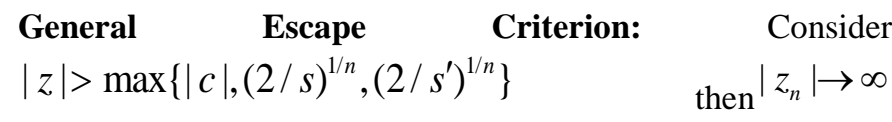
as $n \rightarrow \infty$ is the escape criterion. Note that the initial value $z_{0}$ should be infinity, since infinity is the critical point of $z \rightarrow\left(z^{n}+c\right)^{-1}$. However instead of starting with $z_{0}=$ infinity, it is simpler to start with $z_{1}={ }^{c}$, which yields the same result. (A critical point of $\mathrm{z} \rightarrow \mathrm{F}(\mathrm{z})+\mathrm{c}$ is a point where $\mathrm{F}^{\prime}(z)=0$ ). The role of critical points is explained in [1].

\section{GENERATION OF FRACTALS}

\section{A) Generation Of Fractals For Integer Values:}

4.1 Relative Superior Mandelbrot Sets: We generate Relative Superior Mandelbrot sets. We present here some Relative Superior Mandelbrot sets for quadratic, cubic and biquadratic function.

\subsection{Relative Superior Mandelbrot Sets for} Quadratic function:

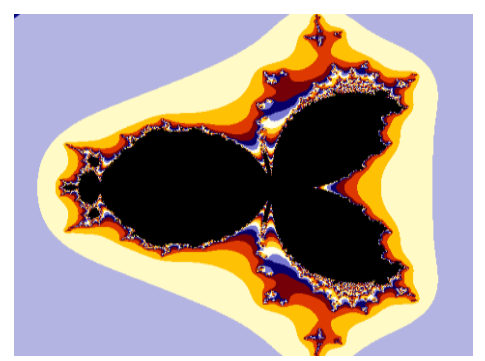

Figure 1: Relative Superior Mandelbrot Set for $s=0.1, s^{\prime}=0.4$

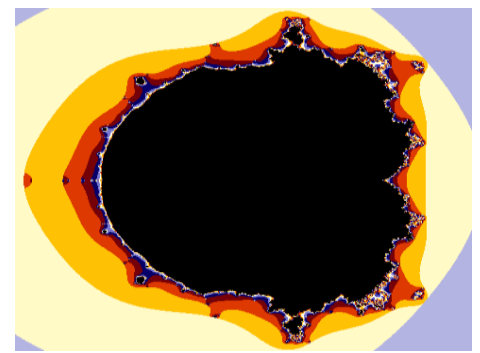

Figure 2: Relative Superior Mandelbrot Set for $s=0.4, s^{\prime}=0.1$

\subsection{Relative Superior Mandelbrot Sets for Cubic function:}

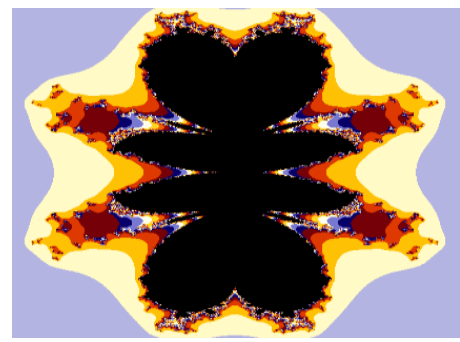

Figure 1: Relative Superior Mandelbrot Set for $s=0.1, s^{\prime}=0.4$

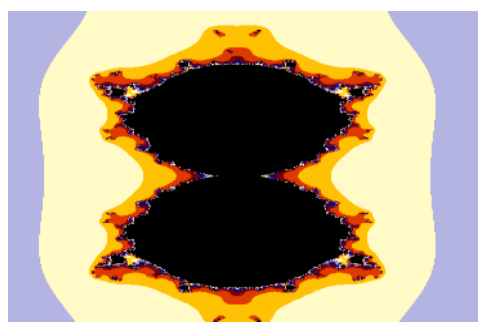

Figure 2: Relative Superior Mandelbrot Set fo

$$
\mathrm{r} \mathrm{s}=0.4, \mathrm{~s}^{\prime}=0.1
$$

\subsection{Relative Superior Mandelbrot Sets for Bi- quadratic function:}

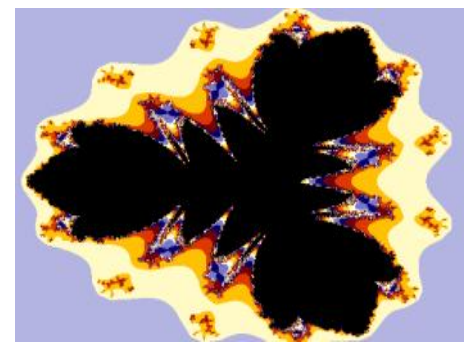

Figure 1: Relative Superior Mandelbrot Set for $s=0.1, s^{\prime}=0.4$ 


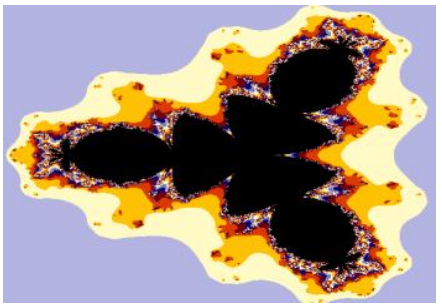

Figure 2: Relative Superior Mandelbrot Set for $s=0.3, s^{\prime}=0.4$

\subsection{Generalization of Relative Superior Mandelbrot} Set

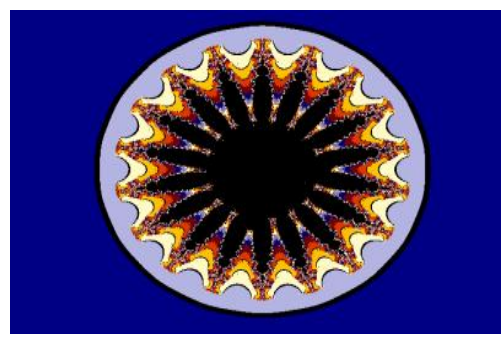

Figure 1: Relative Superior Mandelbrot Set for $s=0.1, s^{\prime}=0.4$, $\mathrm{n}=19$

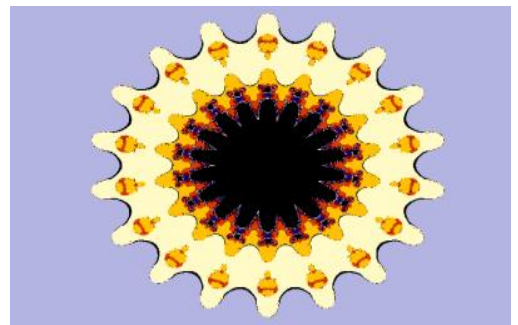

Figure 2: Relative Superior Mandelbrot Set for $s=0.4, s^{\prime}=0.1$, $\mathrm{n}=19$

\subsection{Relative Superior Julia Sets:}

We generate Relative Superior Julia sets. We present here some Relative Superior Julia sets for quadratic, cubic and biquadratic function.

\subsubsection{Relative Superior Julia Sets for Quadratic} function:

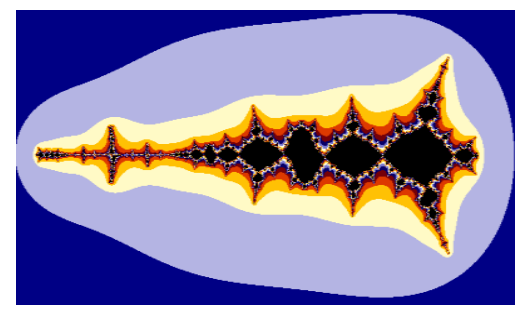

Figure 1: Relative Superior Julia Set for $s=0.1, s^{\prime}=0.4, c=-$ $20.26+0.097 \mathrm{i}$

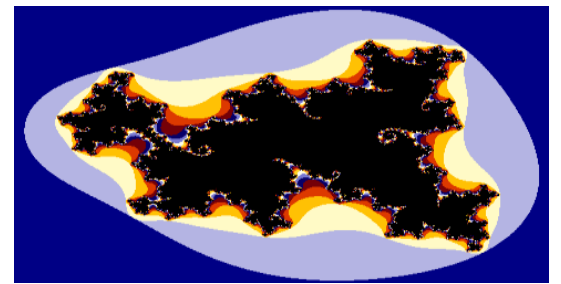

Figure 2: Relative Superior Julia Set for $s=0.4, s^{\prime}=0.1$, $\mathrm{c}=2.1+5.53 \mathrm{i}$

4.22 Relative Superior Julia Sets for Cubic function:

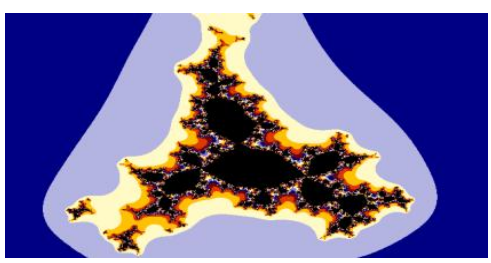

Figure 1: Relative Superior Julia Set for $s=0.1, s^{\prime}=0.4, c=-$ $1.6+6.7 \mathrm{i}$

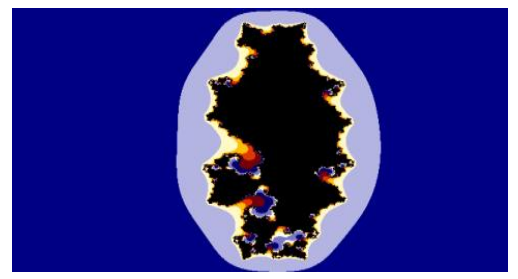

Figure 2: Relative Superior Julia Set for $s=0.4, s^{\prime}=0.1, c=-$ $1+0.5 \mathrm{i}$

4.23 Relative Superior Julia Sets for Bi-quadratic function:

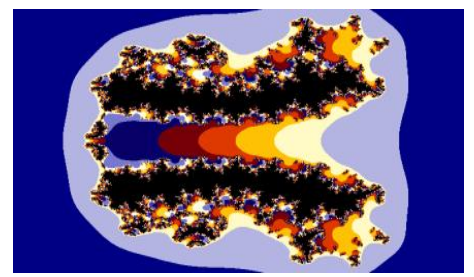

Figure 1: Relative Superior Julia Set for $s=0.1, s^{\prime}=0.4$, $c=2.6+0.0 \mathrm{i}$

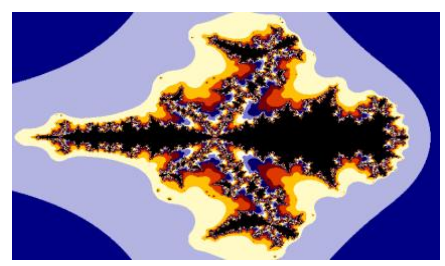

Figure 2: Relative Superior Julia Set for $s=0.3, s^{\prime}=0.4, c=-$ $3.6+0.0 \mathrm{i}$ 


\section{B) Generation Of Fractals For Non-Integer Values:}

4.3 Relative Superior Mandelbrot Sets: We generate Relative Superior Mandelbrot sets. We present here some Relative Superior Mandelbrot sets for quadratic, cubic and biquadratic function.

\subsection{Relative Superior Mandelbrot Sets for Cubic function:}

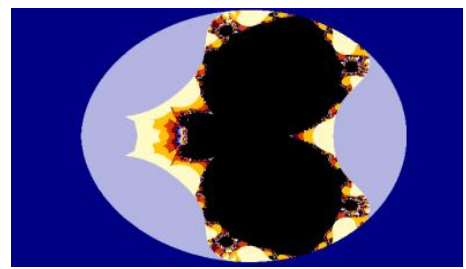

Figure 1: Relative Superior Mandelbrot Set for $\mathrm{s}=0.8, \mathrm{~s}^{\prime}=0.3$, $\mathrm{n}=3.6$

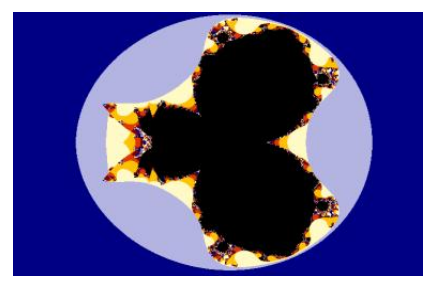

Figure 2: Relative Superior Mandelbrot Set for $\mathrm{s}=0.8, \mathrm{~s}^{\prime}=0.3$, $\mathrm{n}=3.8$

\subsection{Relative Superior Mandelbrot Sets for Bi-} quadratic function:

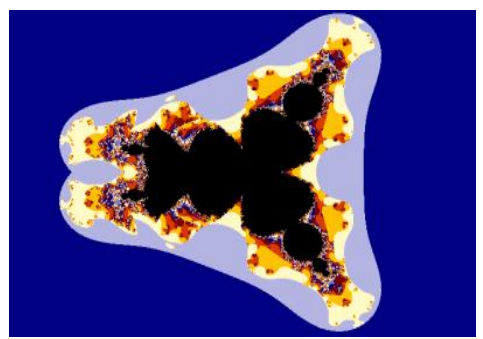

Figure 1: Relative Superior Mandelbrot Set for $\mathrm{s}=0.5, \mathrm{~s}^{\prime}=0.4$, $\mathrm{n}=4.2$

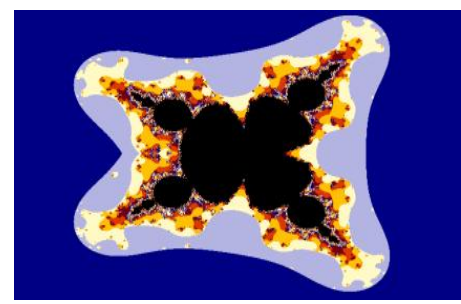

Figure 2: Relative Superior Mandelbrot Set for $s=0.5, s^{\prime}=0.4$, $\mathrm{n}=4.6$
1.33Generalization of Relative Superior Mandelbrot Set:

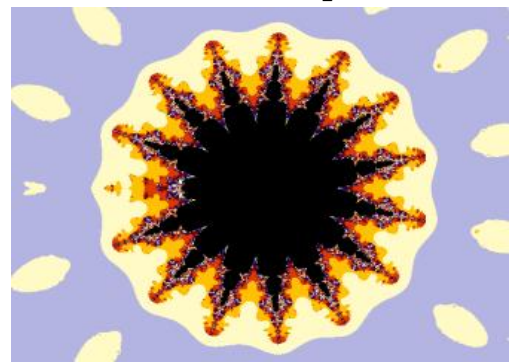

Figure 1: Relative Superior Mandelbrot Set for $\mathrm{s}=0.4, \mathrm{~s}=0.3$, $\mathrm{n}=15.8$

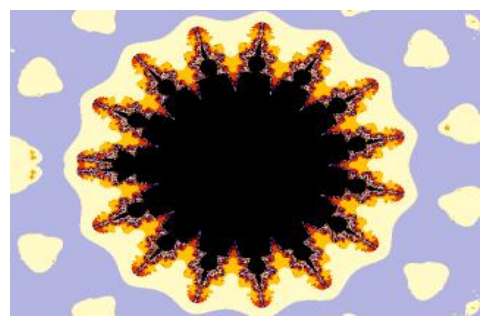

Figure 2: Relative Superior Mandelbrot Set for $\mathrm{s}=0.5, \mathrm{~s}=0.4$, $\mathrm{n}=16.2$

\subsection{RELATIVE SUPERIOR JULIA SETS:}

We generate Relative Superior Julia sets. We present here some Relative Superior Julia sets for quadratic, cubic and biquadratic function.

\subsection{Relative Superior Julia Sets for Cubic function:}

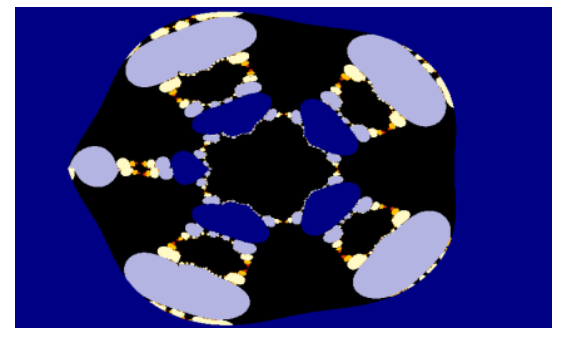

Figure 1: Relative Superior Julia Set for $\mathrm{s}=0.5, \mathrm{~s}^{\prime}=0.4, \mathrm{n}=3.6$, $c=0.03488180321+0.02537719055 \mathrm{i}$

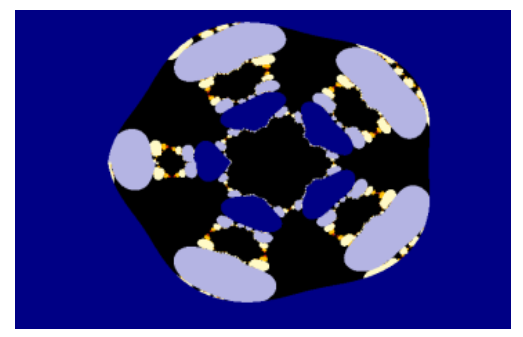

Figure 2: Relative Superior Julia Set for $\mathrm{s}=0.5, \mathrm{~s}^{\prime}=0.4, \mathrm{n}=3.8$, $\mathrm{c}=-0.0442117701+0.03592300032 \mathrm{i}$ 


\subsection{Relative Superior Julia Sets for Bi-quadratic} function:

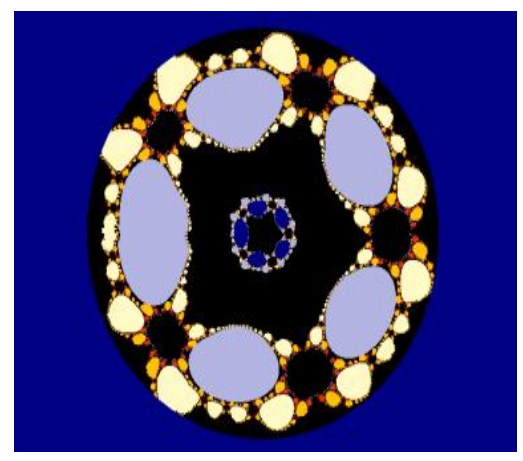

Figure 1: Relative Superior Julia Set $\mathrm{s}=0.8, \mathrm{~s}^{\prime}=0.2, \mathrm{n}=4.2$, $\mathrm{c}=0.04015470798+0.03592299963 \mathrm{i}$

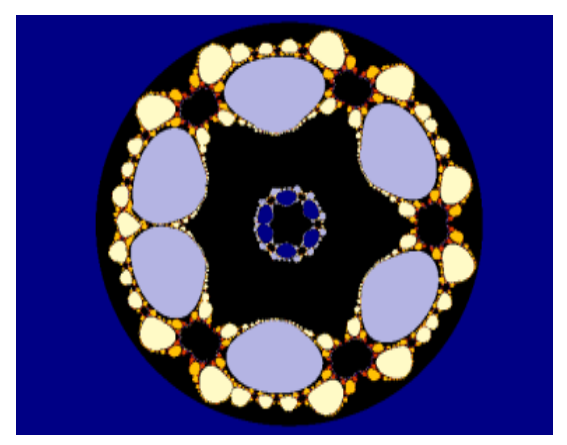

Figure 2: Relative Superior Julia Set for $\mathrm{s}=0.8, \mathrm{~s}^{\prime}=0.2, \mathrm{n}=4.6$, $\mathrm{c}=0.003244373774+0.02010428497 \mathrm{i}$

\section{GRAPHS}

A) Fixed points of Integer values:

\subsection{Fixed points of quadratic polynomial}

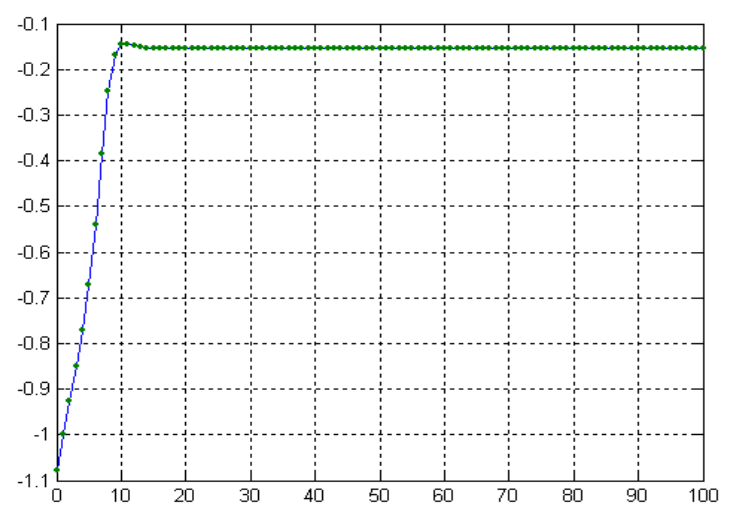

Figure 1: Orbit of $\mathrm{F}(\mathrm{z})$ for $(\mathrm{z} 0=-1.077560973-0.823761912 \mathrm{i}$ ) at $s=0.1$ and $s^{\prime}=0.4$

\subsection{Fixed points of Cubic polynomial :}

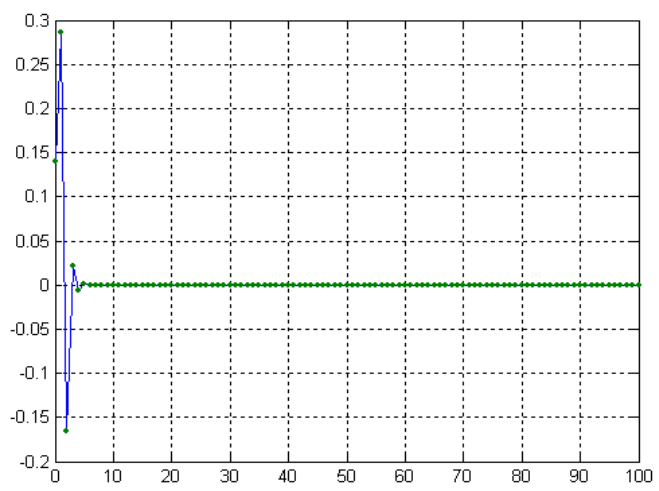

Figure 1: Orbit of $\mathrm{F}(\mathrm{z})$ for $(\mathrm{z} 0=0.14+2.25 \mathrm{i})$ at $\mathrm{s}=0.4$ and $\mathrm{s}^{\prime}=0.1$

\subsection{Fixed points of biquadratic polynomial :}

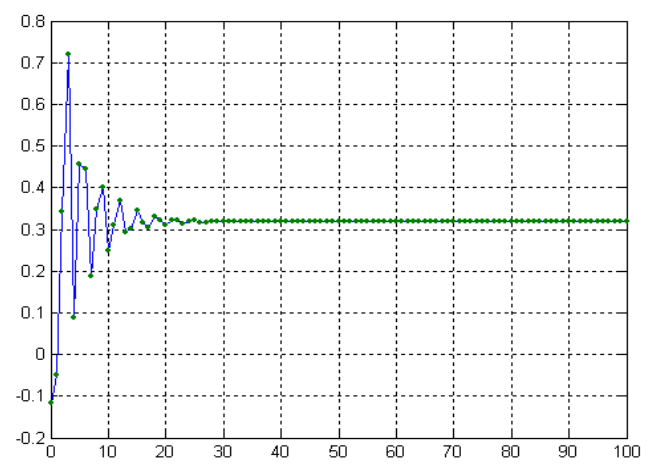

Fig1: Orbit of $\mathrm{F}(\mathrm{z})$ for $(\mathrm{z} 0=-0.118+0.021 \mathrm{i})$ at $\mathrm{s}=0.4$ and $\mathrm{s}^{\prime}=0.1$

B).Fixed points of Non-Integer values:

\subsection{Fixed points of Cubic Polynomial :}

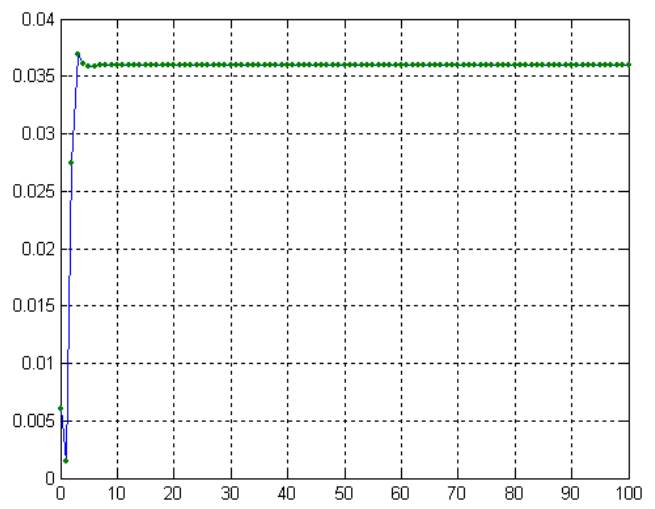

Figure 1: Orbit of $\mathrm{F}(\mathrm{z})$ for $\mathrm{s}=0.8, \mathrm{~s}^{\prime}=0.2, \mathrm{n}=3.6$, $\mathrm{c}=0.006067272682+0$ 


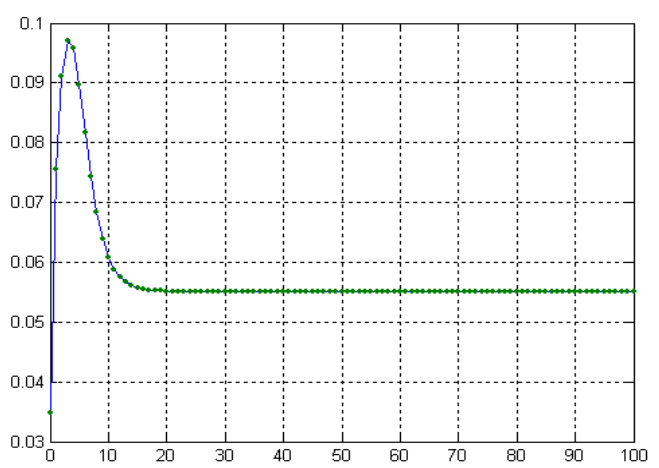

Figure 2: Orbit of $F(z)$ for $s=0.5, s^{\prime}=0.4, n=3.2$, $\mathrm{c}=0.03488180321-0.01680604855$

\subsection{Fixed points of biquadratic polynomial :}

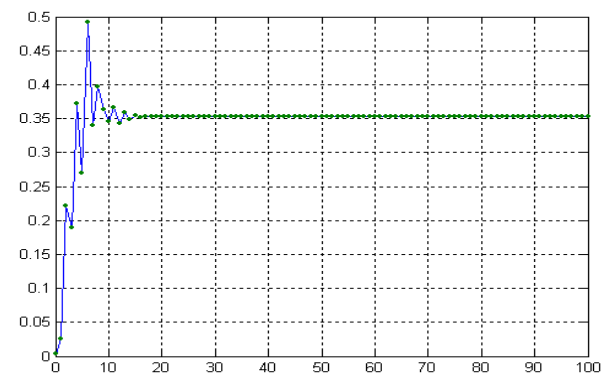

Figure 1: Orbit of $F(z)$ for $s=0.8, s^{\prime}=0.2, n=4.6$, $c=0.003244373774+0.02010428497 \mathrm{i}$

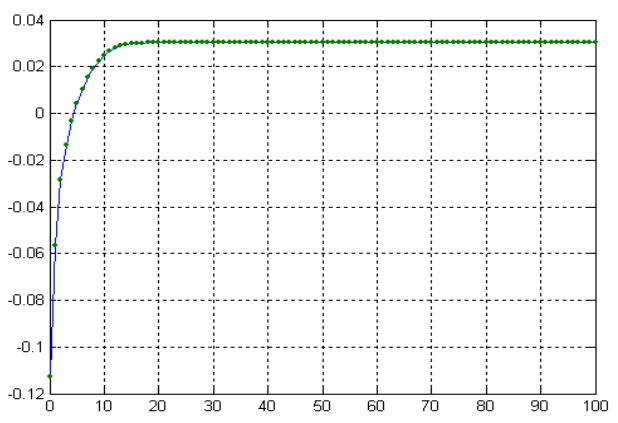

Figure 2: Orbit of $\mathrm{F}(\mathrm{z})$ for $\mathrm{s}=0.5, \mathrm{~s}^{\prime}=0.4, \mathrm{n}=4.8$, $\mathrm{c}=-$ 0.1125374895-0.0520056533i

\section{ANALYSIS}

The $z$ plane fractal images for the function $z_{n+1} \leftarrow z_{n}^{\alpha}+c$ for positive and negative, both integer and non-integer values of $n$ have been presented by Gujar et al. [6, 7] along with some conjectures about their visual characteristics.

$\mathbf{Z}$ plane fractals: The geometrical analysis of Relative Superior Mandelbrot sets for the function $z \rightarrow z^{n}+c, n \geq 2$ reveals the following changes when we move from the positive integer to a positive non integer value:

\section{Relative Superior Mandelbrot Sets:}

- The geometrical analysis of Relative Superior Mandelbrot for the function $z \rightarrow z^{n}+c, n \geq 2$ shows that the stable points of this function in z-plane are black colored. Here the stable region is bounded by the unstable region.

- Also, we notice that the number of lobes in the Relative Superior Mandelbrot sets increases by $(\mathbf{n + 1})$ as one move from an integer to a non integer value nearby the consecutive next integer. Also, for integer values, the symmetry is maintained along real axis for even functions whereas for the odd function, the symmetry is maintained along both axes but such case does not exists for the noninteger terms.

- For $\mathrm{n}=2$, there does not exists Relative Superior Mandelbrot for non-integer function.

- $\quad$ For odd integer $(n=3)$, we see that there are two self similar lobes initially. As we move to $n=3.2$, there exists a small growth between two major lobes, on the left hand side, thus creating asymmetry along the $\mathrm{Y}$ axis, however the symmetry is retained for the $X$ axis. When the value of $n$ increases to 3.6 , we predicted the emergence of a small embryonic lobe. This develops more when we move to $n=3.8$. The generalization $(\mathbf{n}=\mathbf{1 5})$ of this result also presents the same picture of development of embryonic lobe. Thus, the Relative Superior Mandelbrot sets are symmetrical only about the real axis.

- $\quad$ For even integer $(n=4)$, we observe that the size of the major lobe situated on the left hand side (negative axis) is increased. In addition, the major lobe appears to be composed of the two partially overlapping lobes in the form of a composite lobe (At $n=4.2$ ). This composite lobe has two constituent lobes that are visible to us on the verge of splitting two major lobes (At $n=4.6$ ). The generalization of this result is obtained for $\mathbf{n}=\mathbf{1 6}$, where the growth of the embryonic lobe takes place in the same manner. Here also, the Relative Superior Mandelbrot sets are found symmetrical only about the real axis.

C plane fractals: The geometrical analysis of Relative Superior Julia sets for the function $z \rightarrow z^{n}+c, n \geq 2$ reveals the following changes when we move from the positive integer to a positive non integer value:

\section{Relative Superior Julia Sets:}

- Geometrical analysis of the Relative Superior Julia sets of inverse function for non integer values shows that the boundary of the fixed point region forms a $(\boldsymbol{n}+2)$ hypocycloid instead of $(\mathbf{n + 1})$. A hypocycloid is a curve formed by rolling a smaller circle inside a larger circle and tracing a fixed point on the circumference of the smaller circle. The 
radius of the outer fixed circle for hypocycloid can be computed as $|z|+\left|z^{-n}\right|$, where $\mathrm{z}$ satisfies the condition $|z|>n^{1 /(n+2)}$, resulting in a radius of $(n+2) n^{-n /(n+2)}$. The radius of inner moving circle is $\left|z^{-n}\right|$ yielding $n^{-n /(n+2)}$.

- The inverse function for the odd integer $(n=3)$, shows a thin leg appearing at $n=3.6$, which gets broaden as self similar legs at $n=3.8$. So, with the change from the integer to the non integer value the symmetry about both $\mathrm{X}$ and $\mathrm{Y}$ axes, now changes to only about $\mathrm{X}$ axis. Further only the rotational symmetry is maintained while the reflection symmetry is loosed at non integer values.

- The Relative Superior Julia sets for the even integer $(n=$ 4), we observe that the size of the major lobe situated on the left hand side (negative axis) is increased. In addition, the major lobe appears to be composed of the two partially overlapping lobes in the form of a composite lobe (At $n=4.2$ ). This composite lobe has two constituent lobes that are visible to us on the verge of splitting two major lobes (At $n=4.6$ ). Further at $\mathrm{n}=4.8$, an embryonic self similar lobe develops. Thus the symmetry is maintained only along the $\mathrm{X}$ axis for non integer values.

- The generalization of the results is obtained for odd and even values of $n$ can be observed for $n=15$ and $n=16$, where the growth of the embryonic lobe takes place in the same manner. Here the structure resembles to that of a constellation similar to planetary arrangement with central circular planet surrounded by satellite like structures. Furthermore, the size of the central planet reduces as the value of $n$ reduces, while the satellite structures enlarge and diffuses. On the other hand, with the increase in the value of $n$, the central planet size increases and more satellite structures appears with very small radius.

- The planetary arrangement with central circular planet surrounded by satellite like structures describes reflection as well as rotational symmetry.

- It is also observed for the planetary arrangement with central circular planet that, the stable region is represented by light color which surrounds the constellation while the dark color regions represent the unstable areas. This situation is reverse of the situation for the fractals of the function $z \rightarrow z^{n}+c, n \geq 2$ where the stable regions are bounded by unstable regions.

\section{CONCLUSIONS}

In this paper, we have considered the transformation of the function $z \rightarrow z^{n}+c, n \geq 2$. We mathematically analyzed the visual characteristics of the fractal images in the complex $\mathrm{z}$ and $\mathrm{c}$ planes respectively. The $\mathrm{Z}$ plane fractal images for the function $z \rightarrow z^{n}+c, n \geq 2$ showed that the stable region is bounded by unstable region. Besides this, the non integer value change brought the embryonic structure in the form of lobe. On the other hand, the $\mathrm{C}$ plane geometrical analysis of the function $z \rightarrow z^{n}+c, n \geq 2$ represented the planetary type structure comprising of central planet with satellites. Here non integer value change showed the embryonic self similar growth in the satellite pattern. Also, this function exhibits the unstable region embedded within the stable region.

\section{REFERENCES}

[1] B. Branner, "The Mandelbrot Set", Proceedings of Symposia in Applied Mathematics39 (1989), 75-105.

[2] Yashwant S Chauhan, Rajeshri Rana and Ashish Negi. "New Julia Sets of Ishikawa Iterates", International Journal of Computer Applications 7(13):34-42, October 2010. Published By Foundation of Computer Science. ISBN: 978-93-80746-97-5.

[3] Yashwant S Chauhan, Rajeshri Rana and Ashish Negi. Article: "Complex Dynamics of Ishikawa Iterates for Non Integer Values", International Journal of Computer Applications 9(2):9-16, November 2010. Published By Foundation of Computer Science. ISBN: 978-9380747-81-4.

[4] Robert L. Devaney, "A First Course in Chaotic Dynamical Systems: Theory and Experiment", Addison-Wesley, 1992. MR1202237.

[5] S. Dhurandar, V. C. Bhavsar and U. G. Gujar, “Analysis of z-plane fractal images from $z \leftarrow z^{\alpha}+c$ for $\alpha<$ 0”, Computers and Graphics 17, 1 (1993), 89-94.

[6] U. G. Gujar and V. C. Bhavsar, "Fractals from $z \leftarrow z^{\alpha}+c$ in the Complex $c$-Plane", Computers and Graphics 15, 3 (1991), 441-449.

[7] U. G. Gujar, V. C. Bhavsar and N. Vangala, "Fractals from $z \leftarrow z^{\alpha}+c$ in the Complex $z$-Plane", Computers and Graphics 16, 1 (1992), 45-49.

[8] E. F. Glynn, "The Evolution of the Gingerbread Mann”, Computers and Graphics 15,4 (1991), 579-582.

[9] S. Ishikawa, "Fixed points by a new iteration method", Proc. Amer. Math. Soc.44 (1974), 147-150.

[10] G. Julia, "Sur 1' iteration des functions rationnelles", J Math Pure Appli. 8 (1918), 737-747

[11] K. W. Shirriff, "An investigation of fractals generated by $z \rightarrow z^{-n}+c "$, Computers and Graphics 13, 4 (1993), 603-607.

[12] B. B. Mandelbrot, "The Fractal Geometry of Nature", W. H. Freeman, New York, 1983.

[13] H. Peitgen and P. H. Richter, "The Beauty of Fractals", Springer-Verlag, Berlin, 1986.

[14] C. Pickover, "Computers, Pattern, Chaos, and Beauty", St. Martin's Press, NewYork, 1990.

[15] Rajeshri Rana, Yashwant S Chauhan and Ashish Negi. "Non Linear Dynamics of Ishikawa Iteration", International Journal of Computer Applications 
7(13):43-49, October 2010. Published By Foundation of Computer Science. ISBN: 978-93-80746-97-5.

[16] S. T. Welstead and T. L. Cromer, "Coloring Periodicities of Two-dimensional Mappings", Computers and Graphics 13, 4 (1989), 539-543.

\section{BIOGRAPHIES:}

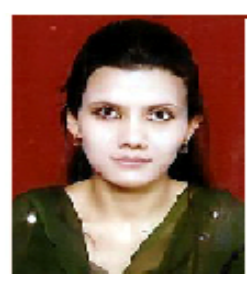

Rajeshri Rana Chauhan is presently serving as Assistant Professor Mathematics in G. B. Pant Enginneeering College, Uttrakhand, India. She is the member of Indian mathematical Society. As an ardent researcher, she is working in the field of fixed point theory and its applications. She has, to her credit many publications in international and national journals and books.

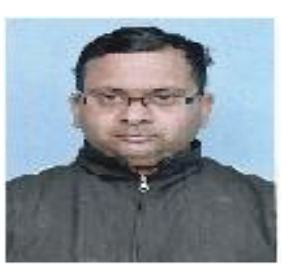

Yashwant Singh Chauhan is presently serving as Assistant Professor Computer Science and Engineering Department in G. B. Pant Enginneeering College, Uttrakhand, India. As an eminent researcher, he is working in the field of Fractals and its applications. He has, to his credit many publications in international journal 